\title{
Message Order Effect in the Context of Multiple Messages in Japan
}

\author{
Masato Nagamine ${ }^{1}$, Miki Toyama ${ }^{1}$, Li Tang${ }^{1}$, Shuhei Miwa ${ }^{2}$, Atsushi Aikawa ${ }^{1}$. \\ ${ }^{1}$ University of Tsukuba/ Center for Research on Educational Testing \\ ${ }^{2}$ Kansai Gaidai University / Center for Research on Educational Testing
}

\begin{abstract}
Order of messages is known to influence the effect of persuasion, which is called the "message order effects." Tormala and Clarkson (2007) investigated the message order effects in the context of multiple messages on different topics, and they posited that the degree of expertise about the prior message was an important factor. They demonstrated that in general, when the expertise about the prior message was high, people evaluated the persuasive effect of the latter (target) message as low. On the other hand, when the expertise about the prior message was low, people evaluated the persuasive effect of the latter message as high. These results suggest that a contrast effect caused by focusing on differences between objects is generally possible in the context of multiple messages. However, the participants in prior studies on this effect have been Westerners. Other studies have indicated different social comparison patterns between Western and Eastern cultures, suggesting the possibility that the contrast effect might not be observed or assimilation effect might be observed instead of the contrast effect in Eastern cultures. This possibility was investigated experimentally in Japanese participants. Results indicated no difference in the evaluation of the latter message based on high or low expertise on the prior message, suggesting neither contrast nor assimilation effects in Japanese participants. This result partly supported our prediction, however, the validity of the expert manipulation of prior messages was low in this study. Therefore, there is a need for further studies.
\end{abstract}

Keywords: assimilation; attitude; contrast; culture; persuasion 